\title{
The classical pion field in a nucleus.
}

\author{
Georges Ripka \\ ECT*, Villa Tambosi, I-38050 Villazano (Trento), ITALY \\ Service de Physique Theorique, Centre d'Etudes de Saclay \\ F-91191 Gif-sur-Yvette Cedex, FRANCE \\ ECT $^{*}-07-20$ preprint
}

November 2, 2018

\begin{abstract}
A self-consistent symmetry arises when the nucleon angular momentum $j$ and the isospin $t$ are coupled to a grand spin $G$. Closed $G$ shells become sources of a classical pion field with a hedgehog shape. Although the amplitude of the pion field, as measured by the chiral angle, is small, it is found to perturb significantly the energies of the nucleon orbits.
\end{abstract}

\section{Introduction.}

The purpose of this paper is to explore some nucleon configurations which give rise to a classical pion field. In most mean field calculations, the symmetries assumed for the intrinsic state do not sustain a classical pseudoscalar pion field. For example, a closed $j$ shell can only give rise to a spherically symmetric mean field. The pseudoscalar classical pion field is expected to be composed of odd- $l$ spherical harmonics so that a closed $j$ shell does not provide a source for the pion field. Nor do the usually assumed deformed intrinsic states of even-even nuclei. This is why only the exchange (Fock) term of the pion exchange interaction contributes to the mean field in these nuclei, while the direct (Hartree) term vanishes.

This is a feature of mean field calculations in which the nucleus is described by a Slater determinant or a quasi-particle vacuum. However, it has been known for decades [1] that a considerable fraction of nuclear binding is due to 2-particle 2-hole excitations caused by the pion exchange interaction. Furthermore, Monte-Carlo calculations of light nuclei suggest that roughly $75 \%$ of the nuclear binding is due to the pion exchange interaction 2. Mean field calculations, which use Skyrme or Gogny forces (see the review [3] and references therein) simulate the 2-particle 2-hole contribution of the pion exchange interaction by an effective density dependent interaction. Relativistic mean field calculations (see the review [4]) simulate the same effect by the presence of a sigma meson. However, the effective interactions (or mesons) used in such 
mean field calculations are not able to explore the possibility that the pion field may favor somewhat different nucleon configurations which give rise to a finite classical pion field.

Such a possibility has however been studied by Sugimoto, Ikeda, Toki et al. [5, 6, 7, 8. They construct an independent particle state composed of either mixed parity nucleon orbits or mixed parity and isospin orbits. The charge and parity of this state are then projected and the energy of the projected state is minimized. They find that the tensor force, which is part of the pion exchange interaction, plays an important role, which is understandable insofar as the projection adds 2-particle 2-hole admixtures to the Hartree-Fock state.

In this work, we investigate a self-consistent symmetry of the pion field which leads us to construct closed $G$-shells where $\vec{G}=\vec{j}+\vec{t}$ is the sum of the angular momentum and isospin of a nucleon orbit.

\section{The non relativistic limit of Weinberg nucle- ons interacting with a chiral field.}

The lagrangian of Weinberg nucleons interacting with a chiral field $U=\exp \left(i \gamma_{5} \theta_{a} \tau_{a}\right)$ is [9]:

$L=\bar{\psi}\left(i \partial_{\mu} \gamma^{\mu}-M+i \gamma^{\mu} U^{\frac{1}{2}}\left(\partial_{\mu} U^{-\frac{1}{2}}\right)\right) \psi+\frac{f_{\pi}^{2}}{4 \nu} \operatorname{tr}\left[\left(\partial_{\mu} U\right)\left(\partial^{\mu} U^{\dagger}\right)\right]+\frac{f_{\pi}^{2} m_{\pi}^{2}}{4 \nu} \operatorname{tr}\left(U+U^{\dagger}\right)$

It can be written in terms of the chiral angle $\theta_{a}$ as follows:

$$
\begin{gathered}
L=\bar{\psi}\left(i \partial_{\mu} \gamma^{\mu}-M\right) \psi \\
+\bar{\psi}\left(-\varepsilon_{a b c} \tau_{a} \sin ^{2} \frac{\theta}{2} \frac{\theta_{b}}{\theta}\left(\gamma^{\mu} \partial_{\mu} \frac{\theta_{c}}{\theta}\right)-g_{A} \gamma_{5} \frac{1}{2} \sin \theta\left(\gamma^{\mu} \partial_{\mu} \frac{\theta_{a} \tau_{a}}{\theta}\right)-g_{A} \gamma_{5} \frac{1}{2} \frac{\theta_{a} \tau_{a}}{\theta}\left(\gamma^{\mu} \partial_{\mu} \theta\right)\right) \psi \\
+\frac{1}{2} f_{\pi}^{2}\left(\left(\partial_{\mu} \theta\right)\left(\partial^{\mu} \theta\right)+\sin ^{2} \theta\left(\partial_{\mu} \frac{\theta_{a}}{\theta}\right)\left(\partial^{\mu} \frac{\theta_{a}}{\theta}\right)-4 m_{\pi}^{2} \sin ^{2} \frac{\theta}{2}\right)
\end{gathered}
$$

where $\psi$ is the nucleon field, $\theta^{2}=\sum_{a=1,3} \theta_{a}^{2}$ and where the last two terms in the second line have been multiplied by the axial coupling constant $g_{A}[9]$. The pion decay constant $f_{\pi}$, the axial coupling constant $g_{A}$, the pion and nucleon masses $m_{\pi}$ and $M$ are respectively:

$$
f_{\pi}=93.2 \mathrm{MeV} \quad g_{A}=1.257 \quad m_{\pi}=139.6 \mathrm{MeV} \quad M=938.9 \mathrm{MeV}
$$

Our results (see Fig (3) yield chiral angles $\theta_{a}$ which do not exceed 0.05 so that we can safely work with the low amplitude limit, in which the chiral field reduces to a pion field. For small values of $\theta_{a}$, the lagrangian (2.2) reduces to:

$$
L=\bar{\psi}\left(i \partial_{\mu} \gamma^{\mu}-M-\frac{1}{2} g_{A} \gamma_{5} \gamma^{\mu} \tau_{a}\left(\partial_{\mu} \theta_{a}\right)\right) \psi+\frac{1}{2} f_{\pi}^{2}\left(\left(\partial_{\mu} \theta_{a}\right)\left(\partial^{\mu} \theta_{a}\right)-m_{\pi}^{2} \theta_{a}^{2}\right)
$$


and the pion field $\pi_{a}$ is related to the chiral angle $\theta_{a}$ by:

$$
\pi_{a}=f_{\pi} \theta_{a}
$$

The mean field approximation consists in assuming that the chiral (or pion) field is classical. We shall further consider time-independent stationary states, in which case we can work with the hamiltonian:

$H=\int d^{3} x \psi^{\dagger}\left(\frac{\vec{\alpha} \cdot \vec{\nabla}}{i}+\beta M-\frac{1}{2} g_{A} \gamma_{5} \tau_{a}\left(\vec{\alpha} \cdot \vec{\nabla} \theta_{a}\right)\right) \psi+\frac{1}{2} f_{\pi}^{2} \int d^{3} x\left(\left(\vec{\nabla} \theta_{a}\right)^{2}+m_{\pi}^{2} \theta_{a}^{2}\right)$

We further proceed to make a non relativistic reduction of the Dirac hamiltonian:

$$
h=\frac{\vec{\alpha} \cdot \vec{\nabla}}{i}+\beta M-\frac{1}{2} g_{A} \gamma_{5} \tau_{a}\left(\vec{\alpha} \cdot \vec{\nabla} \theta_{a}\right)
$$

The reduction is made in two steps. First we maintain the nucleon orbits orthogonal to the vacuum Dirac sea orbits by expanding the eigenstates of $h$ on the positive energy nucleon plane wave states $|\vec{k}, \sigma, \tau,+\rangle$, which are eigenstates of $\frac{\vec{\alpha} \cdot \vec{\nabla}}{i}+\beta M$ belonging to the positive eigenvalues $\sqrt{k^{2}+M^{2}}$. This is achieved by working with a modified Dirac hamiltonian $h_{N R}$ defined such that it has the same matrix elements as $h$ between the positive energy plane wave states:

$$
\left\langle\vec{k}, \sigma, \tau\left|h_{N R}\right| \vec{k}^{\prime}, \sigma^{\prime}, \tau^{\prime}\right\rangle \equiv\left\langle\vec{k}, \sigma, \tau,+|h| \vec{k}^{\prime}, \sigma^{\prime}, \tau^{\prime},+\right\rangle
$$

The second step consists in expanding the resulting $h_{N R}$ in powers of $k / M$. When the expansion is limited to order $k^{2} / M^{2}$ the resulting non relativistic hamiltonian acquires the familiar form:

$$
h_{N R}=-\frac{\nabla^{2}}{2 M}-\frac{g_{A}}{2} \sigma_{i} \tau_{a}\left(\nabla_{i} \theta_{a}\right)
$$

\section{The model hamiltonian and $G$ shells.}

In order to explore nucleon configurations which are likely to give rise to a classical pion field, we further simplify the dynamics by assuming that the nucleons, which interact with the classical pion field, are in spherical oscillator orbits and that they also interact with a spin-orbit contact interaction of the form [3]:

$$
\begin{gathered}
V_{l s}=i W_{l s} \frac{1}{2} \int d^{3} r_{1} d^{3} r_{2} \\
\psi^{\dagger}\left(\vec{r}_{1}\right) \psi^{\dagger}\left(\vec{r}_{2}\right)\left[\varepsilon_{i j k}\left(\sigma_{1}^{i}+\sigma_{2}^{i}\right)\left(p_{1}^{j}-p_{2}^{j}\right) \delta\left(\vec{r}_{1}-\vec{r}_{2}\right)\left(p_{1}^{k}-p_{2}^{k}\right)\right] \psi\left(\vec{r}_{2}\right) \psi\left(\vec{r}_{1}\right)
\end{gathered}
$$

where $\vec{p}=\vec{\nabla} / i$. The expectation value of the spin-orbit interaction $V_{l s}$ in a Slater determinant $|\Phi\rangle$ can be reduced to the form:

$$
\left\langle\Phi\left|V_{l s}\right| \Phi\right\rangle=-4 W_{l s} \int d^{3} r \vec{B}(\vec{r}) \cdot(\vec{\nabla} n(\vec{r}))
$$


where $n(\vec{r})$ and $\vec{B}(\vec{r})$ are the following nucleon densities:

$$
n(\vec{r})=\left\langle\Phi\left|\psi^{\dagger}(\vec{r}) \psi(\vec{r})\right| \Phi\right\rangle \quad \vec{B}(\vec{r})=\left\langle\Phi\left|\psi^{\dagger}(\vec{r})(\vec{\sigma} \times \vec{p}) \psi(\vec{r})\right| \Phi\right\rangle
$$

The model hamiltonian is:

$H=\int d^{3} x \psi^{\dagger}\left(h_{o s c}-\frac{g_{A}}{2} \sigma_{i} \tau_{a}\left(\nabla_{i} \theta_{a}\right)\right) \psi+V_{l s}+\frac{1}{2} f_{\pi}^{2} \int d^{3} x\left(\left(\vec{\nabla} \theta_{a}\right)^{2}+m_{\pi}^{2} \theta_{a}^{2}\right)$

where

$$
h_{o s c}=-\frac{\nabla^{2}}{2 M}+\frac{1}{2} M \omega^{2} r^{2}
$$

We shall diagonalize the single particle hamiltonian in a basis in which the angular momentum $\vec{j}$ and the isospin $\vec{t}$ are coupled to a grand spin $G$ :

$$
|n l j G M\rangle=\sum_{m m_{t}}\left\langle j m, \frac{1}{2} m_{t} \mid G M\right\rangle|n l j m\rangle\left|\frac{1}{2} m_{t}\right\rangle
$$

where $|n l j m\rangle$ is a usual spherical shell model orbit and $\left\langle j m, \frac{1}{2} m_{t} \mid G M\right\rangle$ a Clebsch-Gordan coefficient. In this basis, the single particle spin-orbit interaction remains diagonal. We will show that there is a self-consistent symmetry in which nucleons form closed $G$-shells and the pion field has a hedgehog shape. To any non-zero grand spin and projection $(G, M)$ there correspond four states, which we denote as $|G M i\rangle$ with $i=1,2,3,4$, namely:

\begin{tabular}{|l|l|l|l|}
\hline$|G M 1\rangle$ & $l=G-1=j-\frac{1}{2}$ & $j=l+\frac{1}{2}=G-\frac{1}{2}$ & $P=(-1)^{G+1}$ \\
\hline$|G M 2\rangle$ & $l=G+1=j+\frac{1}{2}$ & $j=l-\frac{1}{2}=G+\frac{1}{2}$ & $P=(-1)^{G+1}$ \\
\hline$|G M 3\rangle$ & $l=G=j-\frac{1}{2}$ & $j=l+\frac{1}{2}=G+\frac{1}{2}$ & $P=(-1)^{G}$ \\
\hline$|G M 4\rangle$ & $l=G=j+\frac{1}{2}$ & $j=l-\frac{1}{2}=G-\frac{1}{2}$ & $P=(-1)^{G}$ \\
\hline
\end{tabular}

To grand spin $G=0$ states correspond two shell model states:

$$
\begin{array}{|l|l|l|l|}
\hline|(G=0), 1\rangle & l=G=0 & j=l+\frac{1}{2}=G+\frac{1}{2}=\frac{1}{2} & P=+ \\
\hline|(G=0), 2\rangle & l=G+1=1 & j=l-\frac{1}{2}=G+\frac{1}{2}=\frac{1}{2} & P=- \\
\hline
\end{array}
$$

For example, the four $|n G M i\rangle$ states with $n=1$ and $G=2$ are:

\begin{tabular}{|l|l|l|l|l|}
\hline$G$ & $i$ & $l$ & $j$ & \\
\hline 2 & 1 & 1 & $\frac{3}{2}$ & $1 p_{\frac{3}{2}}$ \\
\hline 2 & 2 & 3 & $\frac{5}{2}$ & $1 f_{\frac{5}{2}}$ \\
\hline 2 & 3 & 2 & $\frac{5}{2}$ & $1 d_{\frac{5}{2}}$ \\
\hline 2 & 4 & 2 & $\frac{3}{2}$ & $1 d_{\frac{3}{2}}$ \\
\hline
\end{tabular}

and the two states with $n=1$ and $G=0$ are the $1 s_{\frac{1}{2}}$ and $1 p_{\frac{1}{2}}$ states. 


\section{The self-consistent symmetry generated by closed $G$ shells.}

A closed $(n G i)$ shell is a state in which the $2 G+1$ states $|n G M i\rangle$ with different values of $M$ are occupied. Applying Wick's theorem, we find that the expectation value of a tensor operator of rank $K$ and component $q$ in a closed $(n G i)$ shell is

$$
\sum_{M}\left\langle n G M i\left|T_{K q}\right| n G M i\right\rangle=\delta_{K 0} \sqrt{2 G+1}\left\langle n G i\left\|T_{K}\right\| n G i\right\rangle
$$

so that it vanishes unless $K=0$. When applying Wick's theorem, one should remember that the rank $K$ of the tensor refers to rotations in the combined $(\vec{r}, \sigma, \tau)$ space which includes rotations in isospin space. The expectation value, in a closed $G$ shell, of the classical pion field appearing in the hamiltonian (3.4) is:

$$
\mathcal{E}=-\frac{g_{A}}{2} \sum_{M}\left\langle n G M i\left|\tau_{a} \vec{\sigma} \cdot \vec{\nabla} \theta_{a}\right| n G M i\right\rangle
$$

To prevent $\mathcal{E}$ from vanishing, $\tau_{a} \vec{\sigma} \cdot \vec{\nabla} \theta_{a}$ must be a tensor of rank zero in $(\vec{r} \sigma \tau)$ space. Since $\tau_{a}$ is a tensor of rank 1 in this space, the condition for $\mathcal{E}$ not to vanish is that $\vec{\sigma} \cdot \vec{\nabla} \theta_{a}$ should also be a tensor of rank 1 . However, $\vec{\sigma} \cdot \vec{\nabla}$ is a scalar so that the condition requires $\theta_{a}$ to be a vector and this in turn means that the chiral angle must have the so-called hedgehog shape:

$$
\theta_{a}(\vec{r})=\widehat{r}_{a} \theta(r)
$$

where $\widehat{r}=\vec{r} / r$. This also implies that closed $G$-shells have definite parity.

If we calculate $\left(\nabla_{i} \theta_{a}\right)$ with the hedgehog field (4.3), we find:

$$
\sigma_{i} \tau_{a}\left(\nabla_{i} \widehat{r}_{a} \theta\right)=\vec{\sigma} \cdot \vec{\tau} \frac{\theta}{r}+(\widehat{r} \cdot \vec{\sigma})(\widehat{r} \cdot \vec{\tau})\left(\frac{d \theta}{d r}-\frac{\theta}{r}\right)
$$

A lengthy although straightforward calculation will also reveal that the twobody spin orbit interaction $V_{l s}$ gives rise to a single-particle potential of the form:

$$
h_{l s}=4 W_{l s} \frac{1}{r} \frac{d n}{d r} \vec{\sigma} \cdot \vec{L}+4 W_{l s}\left(\frac{1}{r} \frac{d B}{d r}+\frac{B}{r^{2}}\right)
$$

where $\vec{L}=\vec{r} \times \vec{p}$, and where $n(r)$ and $B(r)$ are related to the densities (3.3) as follows:

$$
n(\vec{r})=n(r) \quad \vec{B}(\vec{r})=\widehat{r} B(r)
$$

Each closed $(n G i)$ shell makes the following contribution to these densities:

$n_{n G i}(r)=\sum_{M}\langle n G M i \mid \vec{r}\rangle\langle\vec{r} \mid n G M i\rangle \quad B_{n G i}(r)=-\sum_{M}\langle n G M i \mid \vec{r}\rangle \frac{1}{r} \vec{\sigma} \cdot \vec{L}\langle\vec{r} \mid n G M i\rangle$ 
The total densities $n(r)$ and $B(r)$ are obtained by summing these contributions over the occupied $(n G i)$ shells. Thus the single particle hamiltonian which gives rise to closed $G$ shells is:

$$
\begin{aligned}
h_{N R}=-\frac{\nabla^{2}}{2 M} & +\frac{1}{2} M \omega^{2} r^{2}-\frac{g_{A}}{2}\left[\vec{\sigma} \cdot \vec{\tau} \frac{\theta}{r}+(\widehat{r} \cdot \vec{\sigma})(\widehat{r} \cdot \vec{\tau})\left(\frac{d \theta}{d r}-\frac{\theta}{r}\right)\right] \\
& +4 W_{l s} \frac{1}{r} \frac{d n}{d r} \vec{\sigma} \cdot \vec{L}+4 W_{l s}\left(\frac{1}{r} \frac{d B}{d r}+\frac{B}{r^{2}}\right)
\end{aligned}
$$

The expectation value of the third component $t_{3}=\frac{1}{2} \tau_{3}$ of isospin in a $|n G M i\rangle$ orbit is:

$$
\left\langle n G M i\left|t_{3}\right| n G M i\right\rangle=\frac{M}{2 G}(i=1,4) \quad\left\langle n G M i\left|t_{3}\right| n G M i\right\rangle=-\frac{M}{2(G+1)}(i=2,3)
$$

It follows that a closed $G$ shell has an equal average numbers of neutrons and protons. Furthermore, a closed $G$ shell contains $2 G+1$ orbits and it therefore contains an odd number of nucleons.

\section{Nucleon orbits in a hedgehog field of varying amplitude.}

A self-consistent calculation of the chiral angle requires an initial guess of either the nucleon orbits or of the chiral angle $\theta(r)$. In this respect, a useful guide consists in assuming a simple form for the chiral angle. Since it is a $p$ wave, it likely to grow linearly with $r$ at the origin. It is also likely to decrease exponentially at infinity as $\exp \left(-m_{\pi} r\right)$. The simplest function which will do this is:

$$
\theta(x)=a x e^{-x} \quad x \equiv m_{\pi} r
$$

where $a$ measures the amplitude of the chiral angle. The self-consistent calculation described in section 6 shows that (5.1) is quite a good initial guess for the shape of the chiral angle. An example is displayed in Fig 3 . We can then diagonalize the single particle hamiltonian (4.8) with and plot the spectrum of the nucleon orbits as a function of the amplitude $a$ of the chiral angle (5.1).

In this exploratory calculation, we do not mix radial quantum numbers so that each orbit has a given principal quantum number $n$. The nucleon orbits $|\lambda\rangle$ are thus expanded on a $|n G M i\rangle$ basis:

$$
|\lambda\rangle \equiv\left|n_{\lambda} G_{\lambda} M_{\lambda} k_{\lambda}\right\rangle=\sum_{i} c_{i, k_{\lambda}}^{\left(n_{\lambda} G_{\lambda}\right)}\left|n_{\lambda} G_{\lambda} M_{\lambda} i\right\rangle
$$

The sum over $i$ extends from 1 to 4 when $G>0$ and from 1 to 2 when $G=0$. However, since closed $G$ shells give rise to orbits with definite parity only two 
$|n G M i\rangle$ states contribute to any one orbit $|\lambda\rangle$. The coefficients $c_{i, k_{\lambda}}^{\left(n_{\lambda} G_{\lambda}\right)}$ are determined by diagonalizing the hamiltonian (4.8):

$$
h_{N R}\left|n_{\lambda} G_{\lambda} M_{\lambda} k_{\lambda}\right\rangle=e_{n_{\lambda} G_{\lambda} k_{\lambda}}\left|n_{\lambda} G_{\lambda} M_{\lambda} k_{\lambda}\right\rangle
$$

in this basis. The energy of filled $G$ shells is then the following functional of the chiral angle $\theta$ :

$E(\theta)=\sum_{n_{\lambda} G_{\lambda} k_{\lambda} \in F}\left(2 G_{\lambda}+1\right) e_{n_{\lambda} G_{\lambda} k_{\lambda}}+\frac{1}{2} f_{\pi}^{2} \int d^{3} r\left(\left(\frac{d \theta}{d r}\right)^{2}+\frac{2}{r^{2}} \theta^{2}+m_{\pi}^{2} \theta^{2}\right)+\left\langle\Phi\left|V_{l s}\right| \Phi\right\rangle$

where the last term is the expectation value of the spin-orbit interaction (3.1). The sum is limited to occupied $G$ shells.

Figure 1 shows the spectrum of the low energy nucleon orbits plotted as a function of the amplitude $a$ of the chiral angle. They are calculated using a harmonic oscillator constant $\hbar \omega=12.71 \mathrm{Mev}$ which fits the observed charge radios $2.71 \mathrm{fm}$ of ${ }^{16} \mathrm{O}[10$. Figure 2 shows somewhat higher energy orbits calculated with $\hbar \omega=10.27 \mathrm{MeV}$ which fits the observed charge radius $3.48 \mathrm{fm}$ of ${ }^{40} \mathrm{Ca}$. In both figures the spin-orbit interaction strength was $W_{l s}=15 \mathrm{fm}^{-5}$ adjusted to yield a $5 \mathrm{Mev}$ splitting between the $1 d_{\frac{5}{2}}$ and $1 d_{\frac{3}{2}}$ states in ${ }^{16} O$. When $a=0$ the chiral angle and the pion field vanish and the orbits reduce to spherical shell model orbits $|n l j m\rangle$. In the presence of the chiral field $(a \neq 0)$ each $j$ shell of given parity splits into two $G$ shells with $G=j \pm \frac{1}{2}$.

\section{Self consistent calculation of the chiral angle.}

The chiral angle can be calculated by minimizing the energy (5.4). The equation $\delta E(\theta) / \delta \theta(r)=0$ can be solved for $\theta(r)$ in the form:

$$
\theta(x)=-\frac{m_{\pi}}{2 \pi^{2} f_{\pi}^{2}} \int_{0}^{\infty} x^{\prime 2} d x^{\prime} i_{1}\left(x_{<}\right) k_{1}\left(x_{>}\right) \rho\left(x^{\prime}\right)
$$

where $x_{<}$and $x_{>}$are respectively the lower and upper values of $x$ and $x^{\prime}$ and where the Bessel functions are:

$$
i_{1}(z)=-\frac{\sinh (z)}{z^{2}}+\frac{\cosh (z)}{z} \quad k_{1}(z)=\frac{\pi}{2 z} e^{-z}\left(1+\frac{1}{z}\right)
$$

The source term in 6.1 is calculated in terms of the occupied orbits:

$$
x^{2} \rho(x)=\frac{\delta}{\delta \theta(x)} \sum_{n_{\lambda} G_{\lambda} k_{\lambda} \in F}\left(2 G_{\lambda}+1\right) e_{n_{\lambda} G_{\lambda} k_{\lambda}}
$$

The spectra displayed on Figs 1 and 2 can be used to guess which filled $G$ shells are likely to give rise to a favored configuration. We expect that favored 
configurations will be those which give rise to an appreciable energy gap between the occupied and empty $G$-shells. Once we have decided which $G$ shells are filled, we can make a self-consistent calculation of the chiral angle $\theta(r)$ :

1. We choose a set of closed $G$ shells and, with this set, we calculate the source term $\rho(x)$ using (6.3) with unperturbed orbits.

2. With this $\rho(x)$, we calculate $\theta(x)$ using (6.1).

3 . With this $\theta(x)$ we calculate the orbits (5.2) by diagonalizing the hamiltonian (4.8).

4. With this set of orbits we calculate $\rho(x)$ using (6.3) and we return to step 2. We continue iterating until convergence is reached.

The results are summarized in Tables 1 and 2 for configurations containing $12<A<37$ nucleons and $2 p-1 f$ shell configurations respectively. The tables specify which closed $G$ shells are filled, the number of nucleons, the energy gap separating filled and empty orbits and the maximum value of the chiral angle $\theta(x)$ for each configuration. An orbit labelled, for example, $\left(1 d_{5 / 2}\right) G=2$ refers to an orbit which, on Fig 1, is marked $G=2$ and which converges to the $1 d_{5 / 2}$ state when $a=0$. Although the self consistent orbits are not identical to those plotted on Fig 1, they have a large overlap with the latter. We have only listed cases where the filled $G$ shells have lower energies than the empty shells.

When both $G$ shells emanating from a given $j$ shell are filled, the resulting Slater determinant is equivalent to one in which the $j$ shell is closed and it does not contribute to the source term $\rho(x)$. The closed $j$ shells are listed in the first column of tables 1 and 2. The second column specifies the filled and closed $G$ shells which correspond to partially filled $j$ shells. Since every $G$ shell contains an odd number of nucleons, and since a closed $G$ shell has the same average number of neutrons and protons, configurations which have an odd number of filled $G$ shells refer to odd-even nuclei. Most configurations listed in table 1 are of this type. Thus, for example, the $A=13$ configuration could refer to the mirror nuclei ${ }_{7}^{13} N_{6}$ and ${ }_{6}^{13} C_{7}$ and the $A=31$ configuration to ${ }_{16}^{31} S_{15}$ and ${ }_{15}^{31} P_{16}$.

There is a difference between the energy gaps, displayed in Figs.11and 2, and the energy gaps which are given in Tables 1 and 2, The energy gaps appearing in the figures are plotted as a function of the strength of the pion hedgehog field (as measured by the amplitude of the chiral angle). However, in Tables 1 and 2. the displayed energy gaps are obtained self-consistently by a minimization of the energy. We see that in most odd-even nuclei, the chiral angles which minimize the energy are considerably smaller than the ones which produce the largest energy gaps (displayed in Figs 1 and 2). The reason that the energy required to produce a classical field increases with the amplitude of the chiral angle. This is the case of most odd-even nuclei, listed in Tables 1 and 2, for which the chiral field is not strong enough to produce a level crossing between filled and empty levels.

One exception in Table 1 is the $A=22$ configuration, which has filled $\left(1 d_{5 / 2}\right) \quad G=2$ and $\left(2 s_{1 / 2}\right) \quad G=0$ shells. Fig 1 shows that, for $a<0$, the 
$\left(2 s_{1 / 2}\right) G=0$ orbit crosses the $\left(1 d_{5 / 2}\right) G=3$ orbit and it does so also with the calculated chiral angle. Although it has a low energy gap, this configuration gives rise to a relatively strong chiral field. It refers to an odd-odd nucleus such as ${ }_{11}^{22} N a_{11}$.

Another example is the $A=62$ configuration shown in Table2 In this configuration, three filled $G$ shells become almost degenerate, namely the $\left(1 f_{7 / 2}\right) G=$ $4,\left(2 p_{3 / 2}\right) G=2$ and $\left(2 p_{1 / 2}\right) G=0$ orbits. This is clearly visible in Fig 2 In the self-consistent calculation, they are also almost degenerate, their energies being respectively $53.33,54.68$ and $54.19 \mathrm{MeV}$. This configuration also gives rise to a relatively strong chiral field. It refers to an odd-odd nucleus such as ${ }_{31}^{62} G a_{31}$.

The configurations containing $A=13,15,29,31$ and 37 nucleons in Table 1 and $A=62,65$ and 67 in Table 2 have appreciable energy gaps. For these configurations, the chiral angle reaches maximum values $0.03<\theta_{\max }<0.04$. For the shape $\theta(x)=a x e^{-x}$ this corresponds to $a=e \theta_{\max }$ in the vicinity of \pm 0.1 . In fact, the shape $a x e^{-x}$ is quite similar to the calculated shape of $\theta(x)$ as shown on Fig 3 and the self-consistent single particle spectra are very similar to those which can be read off Figs.1 and 2

\section{Discussion and conclusion.}

In most nuclear structure calculations, the pi-nucleon interaction is included in terms of pion exchange interactions between nucleons and the possibility of generating a classical pion field is usually neglected. In this calculation were have explored such a possibility by constructing independent particle states which generate a hedgehog shaped classical pion field. We have made a parameter free estimate of the generated pion field and we found it to be small: the amplitude of the pion field, as measured by the chiral angle, does not exceed 0.05 . We have found however, that such a classical field can perturb significantly the spectrum of the nucleon orbits. Such effects cannot be detected when Skyrme and Gogny interactions are used or in most relativistic mean field calculations (see the reviews [3] and [4). Indeed, in such calculations, the pi-nucleon interaction is absent and the effects of pion exchange between nucleons is only included in terms of effective interactions (often represented by scalar and vector fields) which simulate pion exchange in the Fock term and 2-particle 2-hole excitations. The present calculation shows that, in the mean field approximation, the pionnucleon interaction does give rise to a classical pion field in even-odd and oddodd nuclei and it points to possible deformations which could be taken into account in, for example, generator coordinate calculations.

We have considered the simplest case of a hedgehog shaped pion field. We have shown that such a field gives rise to nucleon orbits which have grand spin $\vec{G}=\vec{J}+\vec{T}$ and that the hedgehog shape of the pion field is a self-consistent symmetry in nuclei composed of closed $G$ shells (which have $\vec{G}=\vec{J}+\vec{T}=0$ ). Closed $G$ shells are states with equal average numbers of neutrons and protons. A single closed $G$ shell is therefore an even-odd system of nucleons and two 
closed $G$ shells form an odd-odd system.

If such states were stable, they would generate $J=T$ rotational bands, as they do for example in Skyrmions 11] and chiral solitons (see the review [12]). However, because of the weak amplitude of the pion field, the energy gaps created by the pion field in open $j$ shells are not very large and it is unlikely that such rotational bands exist. A rotating nucleus composed of closed $G$ shells would be seriously distorted by Coriolis forces acting on the rotating intrinsic state 13 .

The present calculation does not therefore allow a quantitative prediction of the effect of the classical pion field on the spectra of some odd-even and odd-odd nuclei. It is an exploratory calculation which indicates the kind of deformations that are likely to be important in, for example, generator coordinate calculations of the spectra of odd-even and odd-odd nuclei. We have limited the present calculation to closed $G$ shells which generate a hedgehog shaped pion field. There is no reason why this self consistent symmetry should be favored in all nuclei. Other self-consistent symmetries may be energetically favored when, for example, the neighboring even-even nuclei have quadrupole deformations.

\section{A Some properties of grand spin states.}

Simple expressions may be derived for matrix elements between grand spin states $|n l j G M\rangle$ (3.6). If we adopt the conventions (3.7) and (3.8) to designate the four grand spin states $|G M i\rangle$ which correspond to a given $(G, M)$, then the matrix elements of $\vec{\sigma} \cdot \vec{\tau}$ are:

$$
\begin{gathered}
\left\langle G M i|\vec{\sigma} \cdot \vec{\tau}| G^{\prime} M^{\prime} j\right\rangle=\delta_{G G^{\prime}} \delta_{M M^{\prime}} M_{i j}^{(\sigma \tau)} \\
M_{i j}^{(\sigma \tau)}=\left(\begin{array}{cccc}
1 & 0 & 0 & 0 \\
0 & 1 & 0 & 0 \\
0 & 0 & -\frac{2 G+3}{2 G+1} & \frac{4 \sqrt{G(G+1)}}{2 G+1} \\
0 & 0 & \frac{4 \sqrt{G(G+1)}}{2 G+1} & -\frac{2 G-1}{2 G+1}
\end{array}\right) \quad(G>0), \quad M_{i j}^{(\sigma \tau)}=\left(\begin{array}{cc}
-3 & 0 \\
0 & 1
\end{array}\right) \quad(G=0)
\end{gathered}
$$

The matrix elements of $\vec{\sigma} \cdot \widehat{r}$ are:

$$
\begin{gathered}
\left\langle G M i|\vec{\sigma} \cdot \widehat{r}| G^{\prime} M^{\prime} j\right\rangle=\delta_{G G^{\prime}} \delta_{M M^{\prime}} M_{i j}^{(r \sigma)} \\
M_{i j}^{(r \sigma)}=\left(\begin{array}{cccc}
0 & 0 & 0 & -1 \\
0 & 0 & -1 & 0 \\
0 & -1 & 0 & 0 \\
-1 & 0 & 0 & 0
\end{array}\right) \quad(G>0), M_{i j}^{(r \sigma)}=\left(\begin{array}{cc}
0 & -1 \\
-1 & 0
\end{array}\right) \quad(G=0)
\end{gathered}
$$

The matrix elements of $(\vec{\sigma} \cdot \widehat{r})(\vec{\tau} \cdot \widehat{r})$ are:

$$
\left\langle G M i|(\vec{\sigma} \cdot \widehat{r})(\vec{\tau} \cdot \widehat{r})| G^{\prime} M^{\prime} j\right\rangle=\delta_{G G^{\prime}} \delta_{M M^{\prime}} M_{i j}^{(r \sigma r \tau)}
$$




$$
\begin{gathered}
M^{(r \sigma r \tau)}=\left(\begin{array}{cccc}
\frac{1}{2 G+1} & 2 \frac{\sqrt{(G(G+1))}}{2 G+1} & 0 & 0 \\
2 \frac{\sqrt{(G(G+1))}}{2 G+1} & -\frac{1}{2 G+1} & 0 & 0 \\
0 & 0 & -\frac{1}{2 G+1} & \frac{2}{2 G+1} \sqrt{G(G+1)} \\
0 & 0 & \frac{2}{2 G+1} \sqrt{G(G+1)} & \frac{1}{2 G+1}
\end{array}\right) \quad(G>0) \\
M^{(r \sigma r \tau)}=\left(\begin{array}{cc}
-1 & 0 \\
0 & -1
\end{array}\right) \\
\quad(G=0)
\end{gathered}
$$

The spin-orbit interaction $\vec{L} \cdot \vec{s}$ is diagonal:

$$
\begin{gathered}
\left\langle G M i|\vec{L} \cdot \vec{s}| G^{\prime} M^{\prime} j\right\rangle=\delta_{G G^{\prime}} \delta_{M M^{\prime}} \delta_{i j} A_{i}^{(l s)} \\
A_{i}^{(l s)}=\left(\frac{1}{2}(G-1),-\frac{1}{2}(G+2), \frac{1}{2} G,-\frac{1}{2}(G+1)\right) \quad(G>0) \\
A_{i}^{(l s)}=(0,-1) \quad(G=0)
\end{gathered}
$$

It is sometimes useful to use another grand spin basis $|n l D G M\rangle$. It is

\begin{tabular}{|c|c|c|c|c|}
\hline$|(D) G M 1\rangle$ & $l=G-1$ & $D=1$ & $P=(-1)^{G+1}$ & \multirow{4}{*}{$(G>0)$} \\
\hline$|(D) G M 2\rangle$ & $l=G+1$ & $D=1$ & $P=(-1)^{G+1}$ & \\
\hline$|(D) G M 3\rangle$ & $l=G$ & $D=0$ & $P=(-1)^{G}$ & \\
\hline$|(D) G M 4\rangle$ & $l=G$ & $D=1$ & $P=(-1)^{G}$ & \\
\hline
\end{tabular}
defined by coupling the spin $\vec{s}$ and the isospin $\vec{t}$ to a total spin $\vec{D}=\vec{s}+\vec{t}$ and by coupling the orbital angular momenta $\vec{l}$ and $\vec{D}$ so as to form the grand spin $\vec{G}=\vec{l}+\vec{D}$. For a given $(G, M)$ there are four such states when $G>0$ and two states when $G=0$ :

The grand spin states $|(D) G M i\rangle$ are related to the grand spin states $|G M i\rangle$ as follows:

$$
\begin{aligned}
&|G M 1\rangle=|(D) G M 1\rangle \\
&|G M 2\rangle=|(D) G M 2\rangle \\
&|G M 3\rangle=\sqrt{\frac{G+1}{2 G+1}}|(D) G M 3\rangle+\sqrt{\frac{G}{2 G+1}}|(D) G M 4\rangle \\
&|l j G M 4\rangle=-\sqrt{\frac{G}{2 G+1}}|(D) G M 3\rangle+\sqrt{\frac{G+1}{2 G+1}}|(D) G M 4\rangle \\
&|G=0, i\rangle=|(D) G=0, i\rangle
\end{aligned}
$$




\section{B The contribution of the spin-orbit interaction to the mean field.}

Using the $v(1,2)=v(2,1)$ symmetry of the two body spin-orbit interaction, its second quantized form (3.1) becomes the sum of four terms:

$$
V_{l s}=V^{(1)}+V^{(2)}+V^{(3)}+V^{(4)}
$$

which are:

$$
\begin{gathered}
V^{(1)}=i W_{l s} \varepsilon_{i j k} \int d^{3} r a_{\alpha}^{\dagger} a_{\beta}^{\dagger}\left\langle\alpha\left|\sigma^{i} p^{j}\right| \vec{r}\right\rangle\left\langle\vec{r}\left|p^{k}\right| \delta\right\rangle\langle\beta \mid \vec{r}\rangle\langle\vec{r} \mid \gamma\rangle a_{\gamma} a_{\delta} \\
V^{(2)}=i W_{l s} \varepsilon_{i j k} \int d 1 d 2 a_{\alpha}^{\dagger} a_{\beta}^{\dagger}\left\langle\alpha\left|\sigma^{i}\right| \vec{r}\right\rangle\langle\vec{r} \mid \delta\rangle\left\langle\beta\left|p^{j}\right| \vec{r}\right\rangle\left\langle\vec{r}\left|p^{k}\right| \gamma\right\rangle a_{\gamma} a_{\delta} \\
V^{(3)}=-i W_{l s} \varepsilon_{i j k} \int d 1 d 2 a_{\alpha}^{\dagger} a_{\beta}^{\dagger}\left\langle\alpha\left|\sigma^{i} p^{j}\right| \vec{r}\right\rangle\langle\vec{r} \mid \delta\rangle\langle\beta \mid \vec{r}\rangle\left\langle\vec{r}\left|p^{k}\right| \gamma\right\rangle a_{\gamma} a_{\delta} \\
V^{(4)}=-i W_{l s} \varepsilon_{i j k} \int d 1 d 2 a_{\alpha}^{\dagger} a_{\beta}^{\dagger}\left\langle\alpha\left|\sigma^{i}\right| \vec{r}\right\rangle\left\langle\vec{r}\left|p^{k}\right| \delta\right\rangle\left\langle\beta\left|p^{j}\right| \vec{r}\right\rangle\langle\vec{r} \mid \gamma\rangle a_{\gamma} a_{\delta}
\end{gathered}
$$

In these expressions, $|\alpha\rangle,|\beta\rangle, \ldots$ are nucleon single particle states expressed in an arbitrary basis and a sum is assumed over repeated indices.

We evaluate the expectation value of $V_{l s}$ in the Slater determinant:

$$
|\Phi\rangle=a_{\lambda_{1}}^{\dagger} a_{\lambda_{2}}^{\dagger} \ldots a_{\lambda_{A}}^{\dagger}|0\rangle \equiv \prod_{\lambda \in F} a_{\lambda}^{\dagger}|0\rangle
$$

We use Wick's theorem to obtain:

$$
\begin{gathered}
\left\langle\Phi\left|V_{l s}\right| \Phi\right\rangle \equiv\left\langle\Phi\left|V_{1}\right| \Phi\right\rangle+\left\langle\Phi\left|V_{2}\right| \Phi\right\rangle+\left\langle\Phi\left|V_{3}\right| \Phi\right\rangle+\left\langle\Phi\left|V_{4}\right| \Phi\right\rangle \\
=2 i W_{l s} \sum_{\lambda \mu \in F} \int d^{3} r \varepsilon_{i j k}\left\{\left\langle\lambda\left|\sigma^{i} p^{j}\right| \vec{r}\right\rangle\left\langle\vec{r}\left|p^{k}\right| \lambda\right\rangle\langle\mu \mid \vec{r}\rangle\langle\vec{r} \mid \mu\rangle+\left\langle\lambda\left|\sigma^{i}\right| \vec{r}\right\rangle\langle\vec{r} \mid \lambda\rangle\left\langle\mu\left|p^{j}\right| \vec{r}\right\rangle\left\langle\vec{r}\left|p^{k}\right| \mu\right\rangle\right. \\
\left.-\left\langle\lambda\left|\sigma^{i} p^{j}\right| \vec{r}\right\rangle\langle\vec{r} \mid \lambda\rangle\langle\mu \mid \vec{r}\rangle\left\langle\vec{r}\left|p^{k}\right| \mu\right\rangle-\left\langle\lambda\left|\sigma^{i}\right| \vec{r}\right\rangle\left\langle\vec{r}\left|p^{k}\right| \lambda\right\rangle\left\langle\mu\left|p^{j}\right| \vec{r}\right\rangle\langle\vec{r} \mid \mu\rangle\right\}
\end{gathered}
$$

Using $p^{k}=\frac{1}{i} \nabla^{k}$, integrating by parts and using the antisymmetry of $\varepsilon_{i j k}$ we can reduce $\left\langle\Phi\left|V_{l s}\right| \Phi\right\rangle$ to the form:

$$
\begin{gathered}
\left\langle\Phi\left|V_{l s}\right| \Phi\right\rangle=2 W_{l s} \sum_{\lambda \mu \in F} \int d^{3} r \varepsilon_{i j k}\langle\lambda \mid \vec{r}\rangle\left\langle\vec{r}\left|\sigma^{i} p^{k}\right| \lambda\right\rangle\left(\nabla^{j}\langle\mu \mid \vec{r}\rangle\langle\vec{r} \mid \mu\rangle\right) \\
+2 W_{l s} \sum_{\lambda \mu \in F} \int d^{3} r \varepsilon_{i j k}\langle\mu \mid \vec{r}\rangle\left\langle\vec{r}\left|p^{k}\right| \mu\right\rangle\left(\nabla^{j}\left\langle\lambda\left|\sigma^{i}\right| \vec{r}\right\rangle\langle\vec{r} \mid \lambda\rangle\right) \\
-2 W_{l s} \sum_{\lambda \mu \in F} \int d^{3} r \varepsilon_{i j k}\left\langle\lambda\left|\sigma^{i} p^{j}\right| \vec{r}\right\rangle\langle\vec{r} \mid \lambda\rangle\langle\mu \mid \vec{r}\rangle\left\langle\vec{r}\left|i p^{k}\right| \mu\right\rangle
\end{gathered}
$$




$$
-2 W_{l s} \sum_{\lambda \mu \in F} \int d^{3} r \varepsilon_{i j k}\langle\lambda \mid \vec{r}\rangle\left\langle\vec{r}\left|\sigma^{i} p^{k}\right| \lambda\right\rangle\left\langle\mu\left|i p^{j}\right| \vec{r}\right\rangle\langle\vec{r} \mid \mu\rangle
$$

This expression involves the densities:

$$
\begin{array}{r}
n(\vec{r})=\sum_{\lambda \in F}\langle\lambda \mid \vec{r}\rangle\langle\vec{r} \mid \lambda\rangle \quad \vec{p}(\vec{r})=\sum_{\lambda \in F}\langle\lambda \mid \vec{r}\rangle\langle\vec{r}|\vec{p}| \lambda\rangle \\
\vec{B}(\vec{r})=\sum_{\lambda \in F}\langle\lambda \mid \vec{r}\rangle\langle\vec{r}|(\vec{\sigma} \times \vec{p})| \lambda\rangle \quad \vec{\sigma}(\vec{r})=\sum_{\lambda \in F}\langle\lambda \mid \vec{r}\rangle\langle\vec{r}|\vec{\sigma}| \lambda\rangle
\end{array}
$$

in terms of which:

$$
\left\langle\Phi\left|V_{l s}\right| \Phi\right\rangle=2 W_{l s} \int d^{3} r\left[-\vec{B}(\vec{r}) \cdot(\vec{\nabla} n(\vec{r}))-\vec{p}(\vec{r}) \cdot(\vec{\nabla} \times \vec{\sigma}(\vec{r}))-i \vec{B}(\vec{r}) \cdot \vec{p}(\vec{r})+i \vec{B} \cdot \vec{p}^{*}(\vec{r})\right]
$$

Angular momentum coupling techniques can then readily be used to evaluate the contribution of a closed $G$ shell to these densities, with the result that the densities have the form:

$$
n(\vec{r})=n(r) \quad \vec{p}(\vec{r})=\frac{1}{2 i} \vec{\nabla} n(\vec{r})=\frac{1}{2 i} \widehat{r} \frac{d n}{d r} \quad \vec{\sigma}(\vec{r})=\widehat{r} \sigma(r) \quad \vec{B}(\vec{r})=\widehat{r} \frac{1}{r} B(r)
$$

which could have been be guessed from the fact that closed $G$-shells are invariant under rotations generated by spin + isospin. The second term of (B.7) therefore vanishes. The contribution of the spin orbit interaction to the energy of a closed $G$-shell nucleus reduces thus to:

$$
\left\langle\Phi\left|V_{l s}\right| \Phi\right\rangle=-4 W_{l s} \int d^{3} r \vec{B}(\vec{r}) \cdot(\vec{\nabla} n(\vec{r}))
$$

which is the expression (3.2). The contribution can be expressed in terms of the occupied orbits:

$$
\left\langle\Phi\left|V_{l s}\right| \Phi\right\rangle=-4 W_{l s} \sum_{\lambda \mu \in F} \int d^{3} r\langle\lambda \mid \vec{r}\rangle\left\langle\vec{r}\left|(\vec{\sigma} \times \vec{p})_{i}\right| \lambda\right\rangle\left(\nabla_{i}\langle\mu \mid \vec{r}\rangle\langle\vec{r} \mid \mu\rangle\right)
$$

To obtain the contribution to the mean field, we calculate the (functional) derivative:

$$
\frac{\delta}{\delta\langle\lambda \mid \vec{r} s\rangle}\left\langle\Phi\left|V_{l s}\right| \Phi\right\rangle=-4 W_{l s}\left[\left\langle\vec{r} s\left|(\vec{\sigma} \times \vec{p})_{i}\right| \lambda\right\rangle\left(\nabla_{i} n\right)-\langle\vec{r} s \mid \lambda\rangle\left(\nabla_{i} B_{i}\right)\right]
$$

The contribution of the spin-orbit interaction to the mean field is thus the operator:

$$
h_{l s}=-4 W_{l s}\left(\nabla_{i} n\right)(\vec{\sigma} \times \vec{p})_{i}+4 W_{l s}\left(\nabla_{i} B_{i}\right)
$$

Since $n(\vec{r})=n(r)$, we have:

$$
\left(\nabla_{i} n\right)(\vec{\sigma} \times \vec{p})_{i}=\frac{1}{r} \frac{d n}{d r} \vec{r} \cdot(\vec{\sigma} \times \vec{p})=-\frac{1}{r} \frac{d n}{d r} \vec{\sigma} \cdot \vec{L}
$$




$$
\nabla_{i} B_{i}=\nabla_{i} \widehat{r}_{i} B=\frac{1}{r} \frac{d B}{d r}+\frac{B}{r^{2}}
$$

so that:

$$
h_{l s}=4 W_{l s} \frac{1}{r} \frac{d n}{d r} \vec{\sigma} \cdot \vec{L}+4 W_{l s}\left(\frac{1}{r} \frac{d B}{d r}+\frac{B}{r^{2}}\right)
$$

which is the expression (4.5).

\section{References}

[1] G.E.Brown. Unified theory of nuclear models and forces. North Holland, 1967.

[2] C. Pieper and R.B. Wiringa. Quantum monte carlo calculations of light nuclei. Annual Rev. Nucl. Part. Sci. 51, 2001. nucl-th/0103005.

[3] Michael Bender, Paul-Henri Heenen and Paul-Gerhard Reinhard. Selfconsistent mean-field models for nuclear structure. Rev.Mod.Phys. 75, page $121,2003$.

[4] D.Vretenar, A.V.Afanasjev, G.A.Lalazissis and P.Ring. Relativistic hartree-bogoliubov theory: Static and dynamic aspects of exotic nuclear structure. Phys. Rep. 409, page 101, 2005.

[5] Hiroshi Toki, Satoru Sugimoto, and Kiyomi Ikeda. Relativistic mean-field theory with the pion in finite nuclei. Prog.Theor.Phys. 108, page 903, 2002.

[6] Yoko Ogawa, Hiroshi Toki, Setsuo Tamenaga, Hong Shen, Atsushi Hosaka, Satoru Sugimoto and Kiyomi Ikeda. Chiral sigma model with pion mean field in finite nuclei. Prog. Theor.Phys. 111, page 75, 2004. nucl-th/0312042.

[7] Yoko Ogawa, Hiroshi Toki, Setsuo Tamenaga, Satoru Sugimoto and Kiyomi Ikeda. Charge parity projected relativistic mean field model with pion for finite nuclei. Phys.Rev. C73, page 034301, 2006.

[8] S. Sugimoto, K.Ikeda and H.Toki. Study of the effect of the tensor correlation in oxygen isotopes with the charge and parity projected hartree-fock method. Phys.Rev. C75, page 014317, 2007. nucl-th/0607045.

[9] Steven Weinberg. The Quantum Theory of Fields. Cambridge University Press, 1986.

[10] B.A.Brown, C.R.Bronk and P.E.Hodgson. Systematics of nuclear RMS charge radii. J.Phys.G:Nucl.Phys. 10, page 1683, 1984.

[11] C.R.Nappi G.S.Adkins and E.Witten. Nucl.Phys. B228, page 552, 1983.

[12] Georges Ripka. Quarks Bound by Chiral Fields. Oxford University Press, Oxford, 1997.

[13] J.P.Blaizot and G.Ripka. Phys.Rev. D38, page 1556, 1988. 


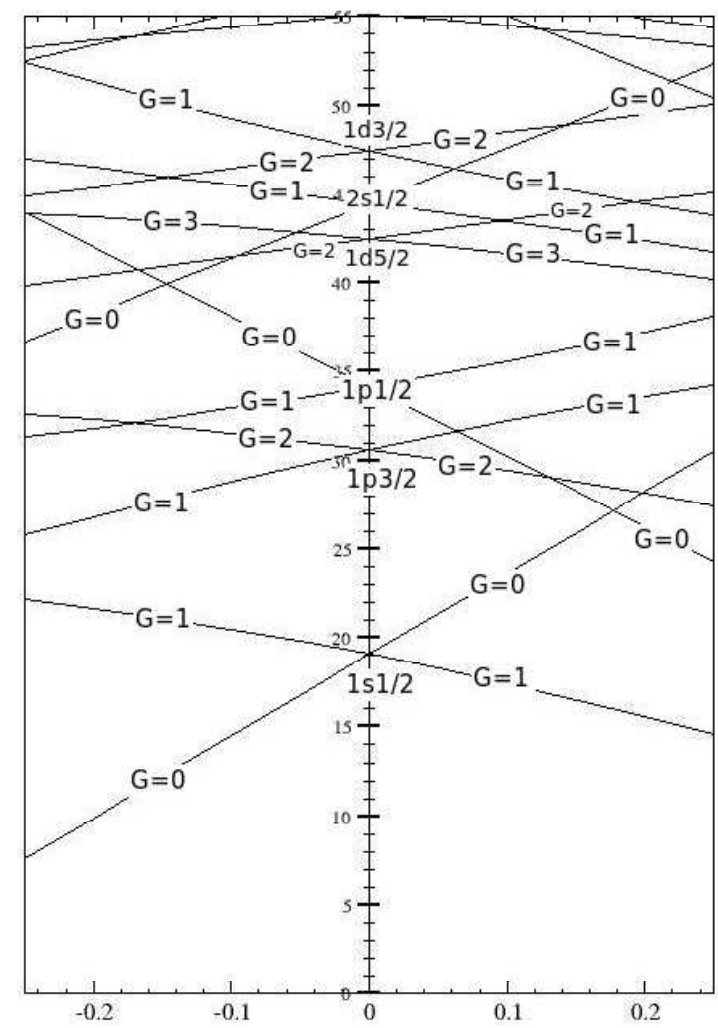

Figure 1: The spectrum of the $|n G M k\rangle$ orbits are plotted as a function of the amplitude $a$ of the chiral angle (5.1). When $a=0$ the chiral angle vanishes and the orbits reduce to spherical shell model orbits $|n l j m\rangle$. When $a \neq 0$ each $j$ shell splits into two $G$ orbits with $G=j \pm \frac{1}{2}$ as indicated on the figure. The orbits were calculated with an oscillator frequency $\hbar \omega=12.71 \mathrm{fm}$ applicable to ${ }^{16} \mathrm{O}$. 


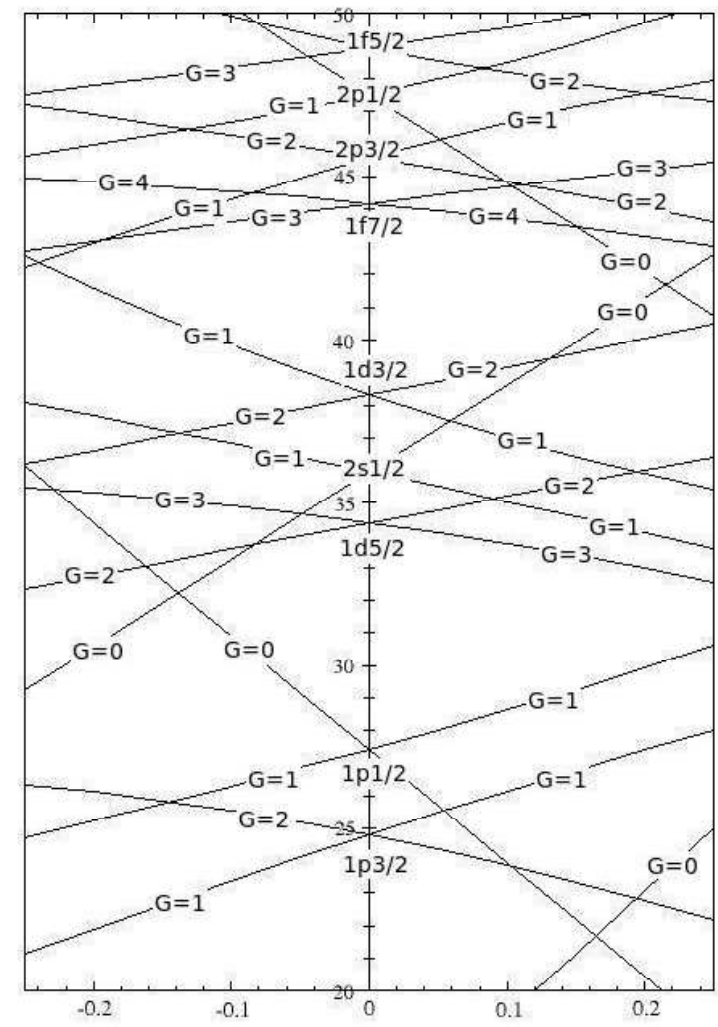

Figure 2: The same as Fig 1 for somewhat higher energy orbits, calculated with $\hbar \omega=10.27 \mathrm{MeV}$ applicable to ${ }^{40} \mathrm{Ca}$. 


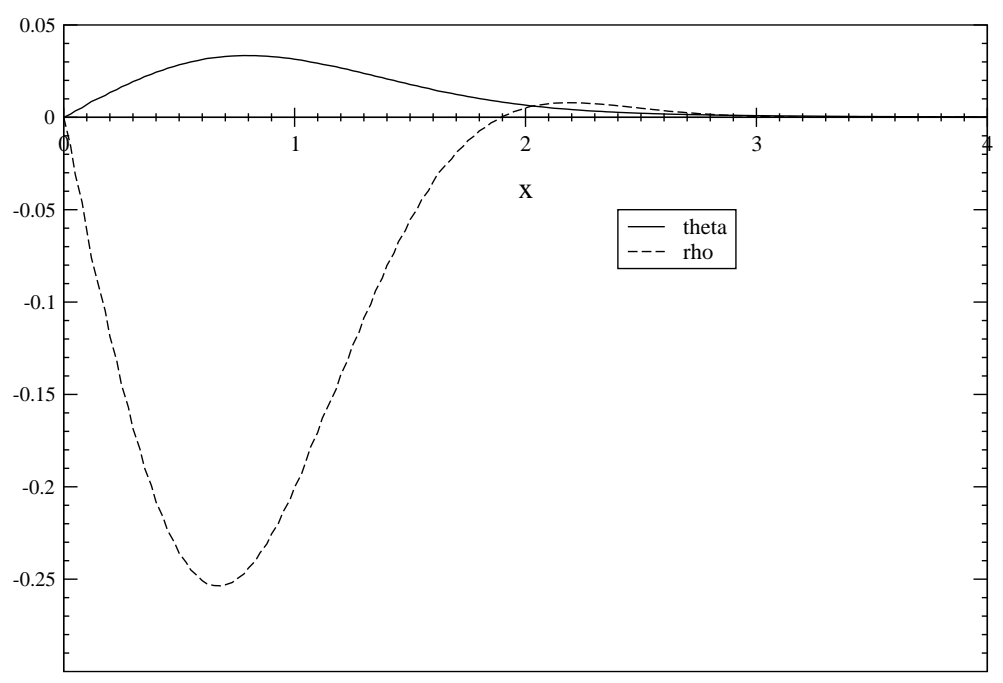

Figure 3: The full line is the chiral angle $\theta(x)$ and the dashed line is the source term $\rho(x)$ defined in (6.3). They are plotted as a function of $x=m_{\pi} r$ for the $A=31$ configuration. The pion field $\pi_{a}(\vec{r})$ can be expressed in terms of $\theta$ as $\pi_{a}(\vec{r})=f_{\pi} \widehat{r}_{a} \theta(r)$. In order to fit the figure, the source term $\rho(x)$ is divided by $4 \pi m_{\pi} f_{\pi}^{2}$. 


\begin{tabular}{|c|c|c|c|c|}
\hline$\hbar \omega=12.71 \mathrm{MeV}$ & & & & \\
\hline Closed $j$ shells & Filled $G$ shells & $A$ & Gap $(\mathrm{MeV})$ & $\theta_{\max }$ \\
\hline $1 s_{1 / 2} G=0,1$ & $\left(1 p_{1 / 2}\right) G=0$ & 13 & 4.14 & 0.038 \\
$1 p_{3 / 2} G=1,2$ & $\left(1 p_{1 / 2}\right) G=1$ & 15 & 4.27 & -0.031 \\
\hline $1 s_{1 / 2} G=0,1$ & & & & \\
$1 p_{3 / 2} G=1,2$ & $\left(1 d_{5 / 2}\right) G=2$ & 21 & 1.17 & -0.017 \\
\hline $1 s_{1 / 2} G=0,1$ & & & \\
$1 p_{3 / 2} G=1,2$ & & & \\
$1 p_{1 / 2} G=0,1$ & & & & \\
\hline $1 s_{1 / 2} G=0,1$ & $\left(1 d_{5 / 2}\right) G=2$ & & & \\
$1 p_{3 / 2} G=1,2$ & $\left(2 s_{1 / 2}\right) G=0$ & & & \\
$1 p_{1 / 2} G=0,1$ & & & & \\
\hline $1 s_{1 / 2} G=0,1$ & & & & \\
$1 p_{3 / 2} G=1,2$ & $\left(1 d_{5 / 2}\right) G=3$ & 23 & & \\
$1 p_{1 / 2} G=0,1$ & & & & \\
\hline $1 s_{1 / 2} G=0,1$ & & & & \\
$1 p_{3 / 2} G=1,2$ & $\left(2 s_{1 / 2}\right) G=0$ & 29 & & \\
$1 p_{1 / 2} G=0,1$ & & & & \\
$1 d_{5 / 2} G=2,3$ & & & & \\
\hline $1 s_{1 / 2} G=0,1$ & & & & \\
$1 p_{3 / 2} G=1,2$ & $\left(2 s_{1 / 2}\right) G=1$ & 31 & 2.92 & 0.03 \\
$1 p_{1 / 2} G=0,1$ & & & & \\
$1 d_{5 / 2} G=2,3$ & & & & \\
\hline$\hbar \omega=10.27 M e V$ & & & & \\
\hline $1 s_{1 / 2} G=0,1$ & & & & \\
$1 p_{3 / 2} G=1,2$ & & & & \\
$1 p_{1 / 2} G=0,1$ & $\left(1 d_{3 / 2}\right) G=1$ & 35 & & \\
$1 d_{5 / 2} G=2,3$ & & & & \\
$2 s_{1 / 2} G=0,1$ & & & & \\
\hline $1 s_{1 / 2} G=0,1$ & & & & \\
$1 p_{3 / 2} G=1,2$ & & & & \\
$1 p_{1 / 2} G=0,1$ & $\left(1 d_{3 / 2}\right) G=2$ & & \\
$1 d_{5 / 2} G=2,3$ & & & & \\
$2 s_{1 / 2} G=0,1$ & & & & \\
\hline
\end{tabular}

Table 1: Closed $G$ shell configurations composed of $12<A<40$ nucleons. The first column lists the filled and closed $j$ shells which do not contribute to the source of the pion field. The second column lists the occupied filled $G$ shells. The column marked $A$ specifies the number of nucleons in the configuration. The last two columns list the energy gap separating filled and empty orbits and the maximum value of the chiral angle $\theta$. 


\begin{tabular}{|c|c|c|c|c|}
\hline$\hbar \omega=10.27 \mathrm{MeV}$ & & & & \\
\hline Closed $j$ shells & Closed $G$ shells & $A$ & Gap $(\mathrm{MeV})$ & $\theta_{\max }$ \\
\hline $1 s, 1 p, 2 s, 1 d$ & $\left(1 f_{7 / 2}\right) G=3$ & 47 & 0.56 & -0.011 \\
\hline $1 s, 1 p, 2 s, 1 d$ & $\left(1 f_{7 / 2}\right) G=4$ & 49 & 0.53 & 0.013 \\
\hline $\begin{array}{c}1 s, 1 p, 2 s, 1 d \\
1 f_{7 / 2} G=3,4\end{array}$ & $\left(2 p_{3 / 2}\right) G=1$ & 59 & 1.58 & -0.029 \\
\hline $\begin{array}{c}1 s, 1 p, 2 s, 1 d \\
1 f_{7 / 2} G=3,4\end{array}$ & $\left(2 p_{3 / 2}\right) G=2$ & 61 & 1.15 & 0.023 \\
\hline $\begin{array}{c}1 s, 1 p, 2 s, 1 d \\
1 f_{7 / 2} G=3,4\end{array}$ & $\begin{array}{c}\left(2 p_{3 / 2}\right) G=2 \\
\left(2 p_{1 / 2}\right) G=0\end{array}$ & 62 & 1.71 & 0.043 \\
\hline $\begin{array}{c}1 s, 1 p, 2 s, 1 d \\
1 f_{7 / 2} G=3,4 \\
2 p_{3 / 2} G=1,2\end{array}$ & $\left(2 p_{1 / 2}\right) G=0$ & 65 & 2.18 & 0.025 \\
\hline $\begin{array}{c}1 s, 1 p, 2 s, 1 d \\
1 f_{7 / 2} G=3,4 \\
2 p_{3 / 2} G=1,2\end{array}$ & $\left(2 p_{1 / 2}\right) G=1$ & 67 & 2.06 & -0.02 \\
\hline
\end{tabular}

Table 2: The same as table1 for $2 p-1 f$ shell nuclei. For all these configurations the $1 s, 1 p, 2 s, 1 d$ shells are filled. 